\title{
Expanding options for RNA based editors
}

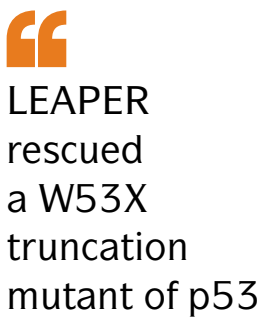

Programmable RNA-guided editing systems have diversified from initial nuclease-based gene editing systems to now encompass break-free base editing of DNA and RNA. Two new studies provide important advances in RNA base editing systems: adenine base editing using unmodified RNA as the only exogenous component, and evolution of an adenine editing system to enable cytosine editing.

Both teams initially worked with adenine base editors in which an ADAR adenine deaminase domain (ADARdd) is fused to a nucleasedeficient Cas13 protein (dCas13). A guide RNA then directs this fusion protein based on complementarity across the RNA target site.

In their study, Qu et al. initially tested how the length of guide RNAs affects RNA base editing efficiency in human cells. They used a two-colour reporter system that requires RNA editing of a stop codon to allow translation of both colours of fluorescent protein from the transcript.

Crucially, the authors noticed that for long guide RNAs of $\sim 70$ nucleotides, the dCas13a-ADAR1dd fusion protein was not required for the base editing. So what was responsible? Editing efficiency was decreased in ADAR1-null cells and increased in ADAR1-overexpressing cells, indicating that the editing was largely

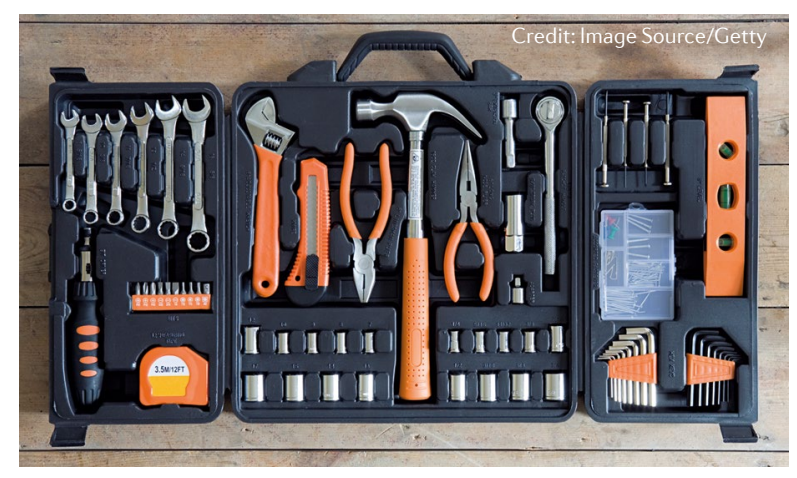

performed by endogenous ADAR1 recruited by the guide RNA.

The main practical implication is that RNA base editing can be achieved without co-delivery of exogenous Cas-based enzymes, which can be challenging to package into common viral vectors and are potentially immunogenic.

This RNA-only approach, termed leveraging endogenous ADAR for programmable editing of RNA (LEAPER) was then optimized for guide RNA length and sequence features. LEAPER showed effectiveness in diverse cell lines and primary cells from humans and mice, via different RNA-delivery routes, and for both exogenous reporter constructs and endogenous RNA targets.

Although further testing is required, the authors found no overt concerns regarding off-target editing rates, unwanted gene expression alterations or immune responses.

As RNA editing is incomplete (transcripts were edited at $~ 5-80 \%$ efficiency across different cell types), it could still be well suited to contexts where no functional protein is produced but where a partial restoration of protein expression could be therapeutic. For proof-of-principle demonstrations in human cells, LEAPER rescued a W53X truncation mutant of $\mathrm{p} 53$ to restore full-length p53 translation and functional p53-mediated transcriptional responses and also rescued defective $\alpha$-L-iduronidase (IDUA) expression in cells from patients with IDUA-defective Hurler syndrome.

In their study, Abudayyeh et al. sought to address the issue that whereas DNA base editor systems have been developed to target either adenines or cytosines, only adenine base editor systems are available for RNA editing. As the human ADAR2dd is structurally similar to
Escherichia coli cytidine deaminase, the authors reasoned that ADAR2dd could be evolved to deaminate cytosines. Starting with Cas13bADAR2dd, they engineered rational mutations in amino acids that form contacts with the target RNA substrate, as well as performing 16 rounds of directed evolution in yeast, whereby ADAR2dd mutants were selected for their ability to edit cytosines in various reporter transcripts. They also rationally optimized the guide RNA.

Overall, the resultant system, termed RNA editing for specific $\mathrm{C}$ to $\mathrm{U}$ exchange (RESCUE) achieved cytosine editing rates of up to $42 \%$ in human cells. This was sufficient for substantial biological effects: editing of the $\beta$-catenin transcript (CTNNB1) to alter a degradation-linked phosphorylation site in the $\beta$-catenin protein led to 5 -fold activation of the signalling pathway and increased cell proliferation.

Furthermore, as the ADAR2dd derivative in RESCUE retains adenine deaminase activity, RESCUE was also directed for multiplexed $\mathrm{C}$ and $\mathrm{A}$ editing in the same transcript using arrays of multiplexed guide RNAs.

Finally, based on assessments of off-target effects, the authors used further rational mutagenesis to create a higher-specificity derivative of RESCUE, termed RESCUE-S.

Both studies are thus important additions to the RNA editing toolbox. For potential therapeutic applications, although RNA editing allays some of the regulatory concerns of permanent genome editing, it will be important to demonstrate that the systems can still provide effects that are sufficiently robust and sustained as to be clinically useful.

Darren J. Burgess

ORIGINAL ARTICLES Qu, L. et al. Programmable RNA editing by recruiting endogenous ADAR using engineered RNAs. Nat. Biotechnol. https:// doi.org/10.1038/s41587-019-0178-z(2019)| Abudayyeh, O. O. et al. A cytosine deaminase for programmable single-base RNA editing. Science 365, 382-386 (2019)

FURTHER READING Rees, H. A. \& Liu, D. R. Base editing: precision chemistry on the genome and transcriptome of living cells. Nat. Rev. Genet. 19, 770-788 (2018) 